\title{
The effect of chlorhexidine mouth wash and visible blue light on Aggregatibacter actinomycetemcomitans and Porphyromonas gingivalis of patients with chronic periodontitis ( an in-vitro study)
}

\author{
${ }^{1}$ Dr. Ali Raad AbdulAzeez, ${ }^{2}$ Dr. Maha Shukri Mahmood, ${ }^{3}$ Dr.Wifaq Mahmood \\ ${ }^{I}$ B.D.S, M.Sc. student. Department of Periodontics. College of Dentistry. University of Baghdad. Baghdad-Iraq. \\ ${ }^{2}$ B.D.S M.Sc. Assistant Professor. Department of Periodontics. College of Dentistry. University of Baghdad. \\ Baghdad-Iraq. \\ ${ }^{3}$ MBchB. ,FICMS. Assistant Professor. College of medicine, unit of infectious clinical diseases. University of \\ Baghdad. Baghdad-Iraq.
}

\begin{abstract}
:
Aims of the study: to determine the effect of $0.2 \%$ chlorhexidine digluconate mouthwash and visible blue light on anaerobic periodontal pathogens namely Aggregatibacter actinomycetemcomitans and Porphyromonas gingivalis.

Materials\& Methods : Strains of Aggregatibacter actinomycetemcomitans and Porphyromonas gingivalis were isolated from pockets of systemically healthy patients aged between 35-55 years old with pocket depths of 5-6 $\mathrm{mm}$, the bacteria cultured on blood Agar plates containing holes filled with $0.1 \mathrm{ml}$ of $0.2 \%$ chlorhexidine, subjected to visible blue light emitted from commercially available light cure devise (LED curing light); that emits blue light (400-500nm) of 1000mw energy at different rates of time exposures, then the inhibition zones of each plate was measured by special ruler after 48 hours of anaerobic incubation.

Results showed that there was an increase in inhibition zone around the chlorhexidine holes, measured by millimeters as we proceed from zero, 20, 40 and 60 seconds of blue light exposure.

Conclusion there is a synergistic effect between visible blue light emitted from the light curing device and $0.2 \%$ chlorhexidine digluconate mouth wash against the anaerobic periodontal pathogens.
\end{abstract}

Key words: Blue light, Chlorhexidine digluconate, anaerobic periodontal pathogen.

\section{Introduction}

Periodontal disease is very common in adults. It results from inflammation of the periodontal tissue around the teeth. Various periodontopathic bacteria cause these periodontal infections ${ }^{1}$. These periodontopathic bacteria exist in the gingival sulcus and periodontal pockets in the biofilm state. Biofilms that colonize the teeth and periodontal pockets are among the most complex biofilms that exist in the ecosystem ${ }^{2}$.

The most frequently used method for periodontal disease treatment is mechanical debridement of biofilm. Nevertheless, it has been demonstrated that mechanical removal of biofilm cannot completely remove all periodontal pathogens from the tooth surface. The anatomical complexity of the roots (furcation areas and root concavities) ${ }^{3,4}$ and bacteria invading the periodontal supporting tissue ${ }^{5-7}$ are among the reasons for this phenomenon.

Accordingly, systemic or local antibiotics have been used to overcome this problem. However, biofilm exhibits several antibiotic-resistance mechanisms ${ }^{8-10}$.The difficulty in maintaining therapeutic antimicrobial concentrations in the oral cavity and disruption of the oral microflora are also problems associated with the use of antibiotics ${ }^{11}$. For these reasons, alternative methods of antimicrobial treatment for periodontal disease are being investigated. Photodynamic therapy (PDT) is one of these alternative methods. PDT involves three indispensable components. These are visible light, a nontoxic photosensitizer, and oxygen ${ }^{12}$. Photosensitizers absorb the visible light that matches the wavelength of their peak absorption, a photochemical mechanism caused by photosensitizers results in bacterial death ${ }^{13-16}$. In most situations, an additional exogenous 
photosensitizer to the target bacteria is required for photosensitization. However, some bacteria do not require exogenous photosensitizers. Black-pigmented bacteria (BPB) indicate the use of an endogenous photosensitizer such as porphyrins, we hypothesize that killing effect is a result of light excitation of their endogenous porphyrins ${ }^{17-19}$.Although classified as anaerobes, the BPB species found in the oral cavity can tolerate low concentrations of oxygen, comparable to those levels in untreated human periodontal pockets. These small amounts of oxygen render periodontal diseases susceptible to Photodynamic therapy (PDT) ${ }^{17}$.

Previous studies have shown that low-energy argon laser irradiation has phototoxic effects on Porphyromonas; Prevotella species ${ }^{19}$.Additionally, visible light has inactivated Porphyromonas gingivalis and Fusobacterium nucleatum without an exogenous photosensitizer ${ }^{19,20}$.

Traditional approaches for reducing the bacterial load include mechanical removal and chemotherapy, the effectiveness of which is compromised by patient motivation, manual dexterity and the development of resistant species ${ }^{21,22}$. In addition, the limited penetration of chemotherapeutic agents into bacterial biofilm results in reduced susceptibility to this kind of treatment ${ }^{23}$. Chemical inhibitors of plaque play an important role in plaque $\operatorname{control}^{24} \cdot \mathrm{A}$ variety of approaches have been considered for chemical plaque control. Most products in current use or under study are antiseptics ${ }^{25}$ Vehicles for delivery of chemical agents with anti-plaque/anti-gingivitis action are toothpastes, mouthwashes, spray, irrigators, chewing gum, and varnishes ${ }^{26}$.However, mouthwashes are a simple and widely accepted method to deliver the anti-microbial agent (after toothpastes), which can be used by the patient as an oral hygiene aid ${ }^{27}$. Chlorhexidine gluconate is an antiseptic mouthwash much in demand. This cationic bis-biguanide is the best known and most widely used member of the class of broad-spectrum antiseptics ${ }^{25}$. It is effective against an array of micro-organisms, including gram-positive and gram-negative organisms, fungi, yeasts, and viruses. It exhibits both anti-plaque and anti-bacterial properties ${ }^{28}$. It acts by altering integrity of cell membrane of bacteria. Its superior anti-plaque activity is the result of its substantively and pin-cushion effect .Any bacteria adhering to the tooth surface are challenged by chlorhexidine molecules, depending on the bacterial species and the amount of chlorhexidine attached to the tooth surface, these micro-organisms are either killed or are simply prevented from multiplying. This bacteriostatic effect of chlorhexidine is what makes it the gold standard. Plaque is prevented from forming because the bacteria attaching the tooth surface cannot multiply. When used as a mouthwash, its mode of action is purely topical and because it is poorly absorbed systemically, it is regarded as a relatively safe drug ${ }^{25}$.

\section{Materials and methods}

Patients selection and Sampling :On day 1, systemically healthy patients of age range between 35-55 years old were chosen, they had chronic periodontitis with at least 4 sites with pocket depth of $4 \mathrm{~mm}$ or greater. Pockets of $5-6 \mathrm{~mm}$ were chosen, tooth was cleaned by spray of water then dried with cotton, and area was isolated with cotton rolls to prevent contamination from saliva and adjacent soft tissue.

Periodontal gracey curettes were used to excavate a piece of plaque from the depth of pocket, the procedure must be done very carefully to avoid touching adjacent tissue to maintain pure subgingival plaque and transferred directly on a swap into a transport media .

$\mathbf{1}^{\text {st }}$ culture: Plaque sample was spread within less than 10 minutes on specific media plates for A.a \& P.gingivalis in a sterile hood. Plates were securely transported into a well-sealed anaerobic jar with anaerobic gas bag (commercially available ready-made oxon ${ }^{\mathrm{TM}}$ bag that is activated automatically after removal of aluminum foil, and leaving the inner sachet inside the jar and the jar is sealed immediately).The anaerobic gas bag will generate $5 \% \mathrm{CO} 2,90 \% \mathrm{~N} 2$ and $5 \% \mathrm{H} 2$, gradually oxygen was deprived from inside the jar generating a negative pressure that sensed when opening the jar seal with resistance. The anaerobic jar was kept in incubator of $37^{\circ} \mathrm{C}$ for $48-72$ hours.

First subculture and bacterial Identification: On day 4, after 72 hours of incubation, bacteria selection was based on colony morphology( shape and size \& color), Gram tests, biochemical activity tests like catalyse test, hemolytic capability, urease test, antibiotic sensitivity to Clindamycin,Vancomycin, Kanamycin \&Metronidazole tests and microscopic appearance). Aggregatibacter actinomycetemcomitans colonies showed a convex white starry appearance with no black pigmentation they were Gram negative with rod shaped appearance under microscope, catalyse positive, coagulase negative, urease negative, had an obvious hemolytic activity and were resistant to Clindamycin and Metronidazole .Porphyromonas gingivalis colonies were dull 
colored round convex colonies, clearly distinguished by the presence of black pigmentation, they were Gram negative with rod shaped (sometimes encapsulated) under microscope, catalyse negative, urease negative, had a weaker hemolytic activity, and susceptible to clindamycin and metronidazole but resistant to kanamycin (Fig $1 \&$ 2).

Second subculture: On day 7, The chosen colonies then were subculture again anaerobically for 72 hours under the same condition, using the same method, to obtain pure cultures of both Aggregatibacter actinomycetemcomitans and Porphyromonas gingivalis.

Serial dilution, application of CHX and light exposure: On day 10, after incubation period, a serial dilution procedure was performed for standardization of the amount of bacteria using $10^{6} \mathrm{CFU}$ as initial concentration to be diluted by using a thioglycolate broth in micro titer plate (Fig 3).Under sterile condition, a micropipette was used to obtain a standard volume of thioglycolate broth to be dispersed in each well, $(150 \mu \mathrm{l})$, then a single colony of each micro-organism was carefully chosen and mixed into the well of broth, from that well, we took $15 \mu \mathrm{l}$ using a micropipette and dispersed it into $135 \mu \mathrm{l}$ of fresh broth, we repeated this procedure for 5 consecutive times to obtain 5 (1:10) dilutions.

We covered the micro titer with liquid paraffin \& sealed by parafilm to ensure the anaerobic conditions and incubate it for an hour in the incubator. For each micro-organism, four plates of fresh enriched blood agar of selective media were prepared; spreading $5 \mathrm{ul}$ of broth taken from the last dilution on the plate in a cross mattress pattern for all four plates. A hole at the center of plate was made by a sterile pasture pipette, to ensure the size of hole was standardized for all experiments. Then $0.01 \mathrm{ml}$ of $0.2 \%$ CHX mouthwash was dispersed carefully in the central holes after light exposure. Each plate was exposed to different times of light exposure, a light beam of blue light $(400-500 \mathrm{~nm})$ of $1000 \mathrm{mw}$ energy was directed on the plate, the center of light beam was directed towards the center of the plate and the diameter of light zone covers the entire plate for standardization.

starting from zero/seconds(no light exposure) for the first plate, then 20,40,60 for the $2^{\text {nd }} 3^{\text {rd }}$ and $4^{\text {th }}$ plates respectively; tip of the light cure device was standardized at $5 \mathrm{~cm}$ distance from the center of plate with the center of light beam was directed towards the center of plate for all experiments. All the plates were transported very carefully into anaerobic gas bag and incubated again under anaerobic conditions at $37 \mathrm{c}^{\circ}$. After 72 hours of incubation, the plates were taken out from the anaerobic jar $\&$ the colonies were noticed around the hole to observe the changes in the $\mathrm{CHX}$ inhibition zone.

Measuring the inhibition zones: On day 13, the inhibition zones were measured by millimeters, starting from the edge of the CHX hole to the nearest colony at the circumference using a small ruler. The plate that has no light exposure (zero seconds group) for each micro-organism considered the control plate with which we compared the results of the remaining 3 plates. The whole procedure was repeated for each one of the 12 patients we have chosen.

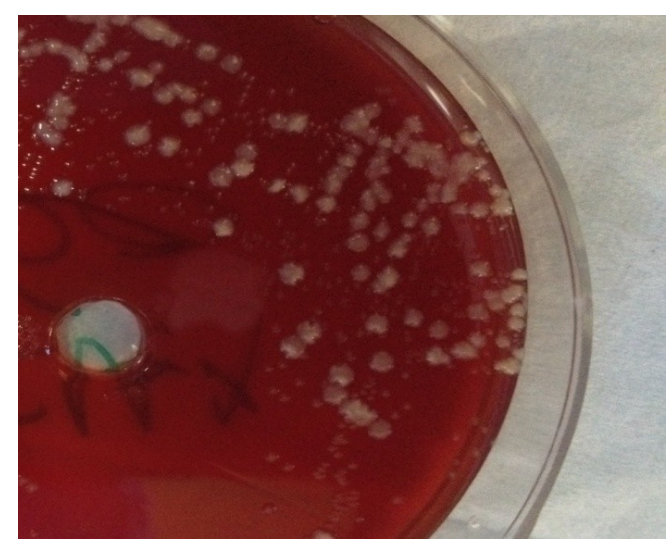

Fig 1

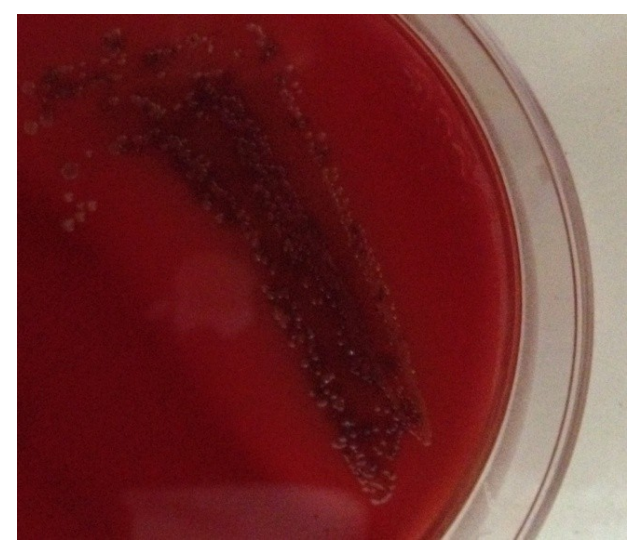

Fig 2

Fig 1: Aggregatibacter actinomycetemcomitans with its white starry shape

Fig 2: Porphyromonas gingivalis with black pigmentation clearly seen on plate 


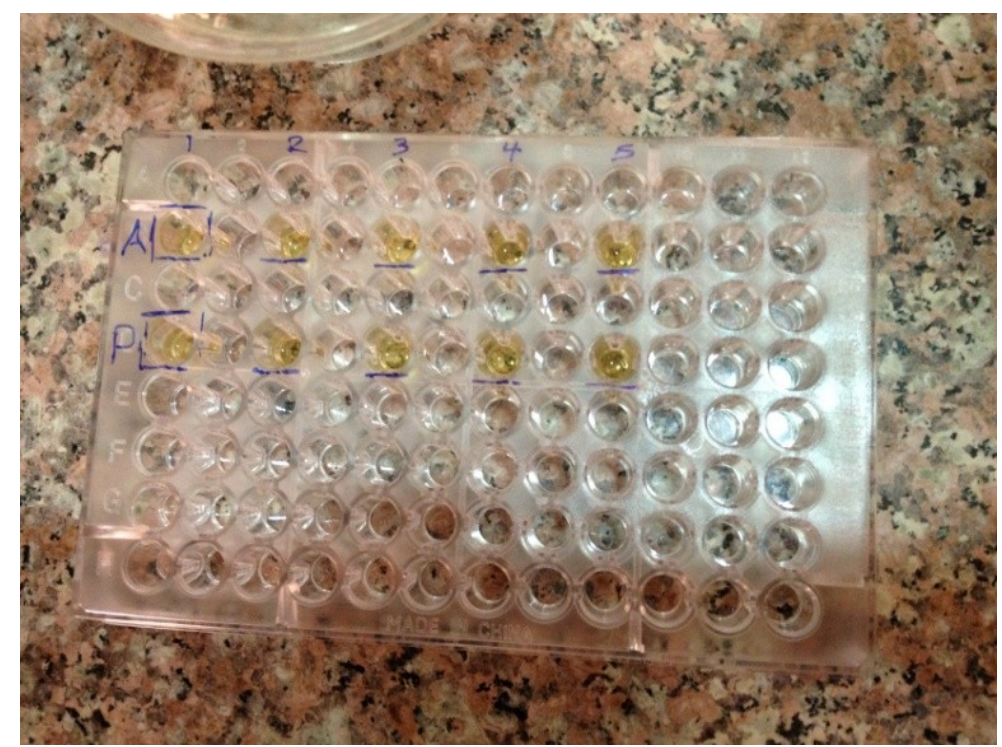

Fig 3: a microtiter plate used for serial dilution and standardization of bacteria in fixed amount of broth

\section{Results}

Plaque samples obtained from different depths of pockets showed variety in the continent.

Several pilot studies were made to standardize the choice of pocket depth, the time of incubation and the subculture procedures, after taking plaque samples from $4 \mathrm{~mm}, 5 \mathrm{~mm}$ and $>5 \mathrm{~mm}$ pocket depths, there was no difference in the outcome between pocket depths of $5 \mathrm{~mm}$ and greater but samples taken from pocket depth of $4 \mathrm{~mm}$ was deprived from the Aggregatibacter actinomycetemcomitans, the number was too little and the characteristics of micro-organisms were not clear or controversial.

Rate of Aggregatibacter actinomycetemcomitans and Porphyromonas gingivalis increased when the pocket was equal or greater than $5 \mathrm{~mm}$. During the first 2 days of incubation, colonies of Aggregatibacter actinomycetemcomitans and Porphyromonas gingivalis didn't show the typical gram negative microscopic appearance, the gram negative characteristic increased to reach nearly absolute on day 3.Same thing can be applied on colony morphology, clearer shape and more typical picture was obtained on day 3.Strains of Porphyromonas gingivalis varied in the production of $\mathrm{H} 2 \mathrm{~S}$ which is responsible for black pigmentation. Some strains derived from different patients showed more black pigmentation than others, in other strains, daughter bacteria were weaker after subculture regarding the colony size, and presence of black pigmentation. Strains derived from different patients also varied in their response to light exposure, some strains were more resistant than others regarding colonies size, and number which increased as we proceeded further from the center of light exposure and the central hole that contained the chlorhexidine. When Aggregatibacter actinomycetemcomitans was exposed to air, the colonies gained darker color and lost their distinguished shape.

A significant statistical difference was observed in comparing the median value of CHX mouthwash inhibition zones against Aggregatibacter actinomycetemcomitans at different periods of blue light exposure(table 1)(Fig 4), there was a gradual increase in the CHX inhibition zones; as we proceeded from zero to 60 seconds of blue light exposure (Fig 5), (Fig 6 A\& B).In intergroup comparison between each of the periods of light exposure time, there was a significant statistical difference between the control group (had no light exposure) and the 60 second group(Table 2).

Concerning the Porphyromonas gingivalis, there was also a significant statistical difference in comparing the median values of CHX mouthwash inhibition zones against Porphyromonas gingivalis at different periods of blue light exposure(table 3)(fig 7), there was an increase in the CHX inhibition zones between zero and 60 seconds of blue light exposure (Fig 8 A\& B).In intergroup comparison between each of the periods of light exposure time, there was a significant statistical difference between the control group (had no light exposure) and the 60 second group(Table 4). 


\section{Discussion}

In the last century numerous evidences have emerged supporting the concept that, in susceptible hosts, bacteria cause periodontal diseases ${ }^{29}$. The antibacterial effect of chlorhexidine was mentioned by many authors and researchers, At low concentration, it causes increase cell permeability with leakage of intracellular components including potassium. At high concentration, chlorhexidine causes precipitation of bacterial cytoplasm and cell death $\&$ it is significantly reduced plaque growth \& gingival inflammation ${ }^{30,31}$.

As it was observed from the experiment, the effect of chlorhexidine was enhanced when the plate is subjected to blue light. This can be seen clearly by the increase of CHX inhibition zone when compared to CHX inhibition zone without light exposure .The inhibition zones increased more as the period of blue light exposure increased.

Researchers suggested the presence of phototoxic effect of blue light on anaerobic periodontal pathogens with several explanations. Visible light $(408-750 \mathrm{~nm})$ has been found to be mutagenic and cause metabolic and membrane damage to bacteria such as Escherichia coli. Feuerstein et al. 2004 suggested that increases in temperature could damage bacteria after exposure to blue light. Oxidative stress occurs with reactive oxygen species such as superoxide anion, hydrogen peroxide, and hydroxyl radicals that damage proteins, DNA, lipid, and the cell membrane ${ }^{32}$.Hyun-Hwa Song et al. (2013) showed that blue light exposure is available to reduce periodontal pathogens in the planktonic state. However, periodontal pathogens in the intraoral environment exist in a biofilm state. Blue light exposure to periodontal pathogens in the biofilm state was less effective than in the planktonic state. And found a Little phototoxic effect even in the biofilm states of A. actinomycetemcomitans and F. nucleatum. Therefore he recommended that an adjunctive exogenous photosensitizer (e.g., methylene blue, toluidine blue $\mathrm{O}$, erythrosine) be used when visible light exposure is used for antimicrobial periodontal therapy ${ }^{17}$.

\section{Conclusion}

There was a synergistic effect between chlorhexidine mouth wash and visible blue light $(400-500 \mathrm{~nm})$ of $1000 \mathrm{mw}$ energy in vitro on anaerobic periodontal pathogen (Aggregatibacter actinomycetemcomitans and Porphyromonas gingivalis).

\section{References}

[1]. Darveau RP, Tanner A, Page RC: The microbial challenge in periodontitis, Periodontol 2000 14:12- 22, 1997

[2]. Fontana CR, Abernethy AD, Som S, Ruggiero K, Doucette S, Marcantonio RC, et al. The antibacterial effect of photodynamic therapy in dental plaque-derived biofilms. J Periodontal Res. 2009; 44:751-759.(Taken from IVSL)

[3]. Adriaens PA, Adriaens LM. Effects of nonsurgical periodontal therapy on hard and soft tissues. Periodontol 2000. 2004;36:121145. (Taken from IVSL)

[4]. Umeda M, Takeuchi Y, Noguchi K, Huang Y, Koshy G, Ishikawa I. Effects of nonsurgical periodontal therapy on the microbiota. Periodontol 2000. 2004;36:98-120. [PubMed]

[5]. Meyer DH, Sreenivasan PK, Fives-Taylor PM. Evidence for invasion of a human oral cell line by Actinobacillus actinomycetemcomitans. Infect Immun. 1991;59:2719-2726.

[6]. Amano A. Disruption of epithelial barrier and impairment of cellular function by Porphyromonas gingivalis. Front Biosci. 2007; 12:3965-3974.

[7]. Thiha K, Takeuchi Y, Umeda M, Huang Y, Ohnishi M, Ishikawa I. Identification of periodontopathic bacteria in gingival tissue of Japanese periodontitis patients. Oral Microbiol Immunol. 2007;22:201-207.

[8]. Fux CA, Costerton JW, Stewart PS, Stoodley P. Survival strategies of infectious biofilms. Trends Microbiol. 2005;13:34-40.

[9]. Del Pozo JL, Patel R. The challenge of treating biofilm-associated bacterial infections. Clin Pharmacol Ther. 2007;82:204-209.

[10]. Anderson GG, O'Toole GA. Innate and induced resistance mechanisms of bacterial biofilms. Curr Top Microbiol Immunol. 2008;322:85-105.

[11]. Wilson M. Lethal photosensitization of oral bacteria and its potential application in th photodynamic therapy of oral infections. Photochem Photobiol Sci. 2004;3:412-418.

[12]. Takasaki AA, Aoki A, Mizutani K, Schwarz F, Sculean A, Wang CY, et al. Application of ntimicrobial photodynamic therapy in periodontal and peri-implant diseases. Periodontol 2000. 2009;51:109-140.

[13]. Meyer DH, Sreenivasan PK, Fives-Taylor PM. Evidence for invasion of a human oral cell line by Actinobacillus actinomycetemcomitans. Infect Immun. 1991;59:2719-2726.

[14]. Sharman WM, Allen CM, van Lier JE. Photodynamic therapeutics: basic principles and clinical applications. Drug Discov Today. 1999;4:507-517.

[15]. Maisch T, Szeimies RM, Jori G, Abels C. Antibacterial photodynamic therapy in dermatology. Photochem Photobiol Sci. 2004;3:907-917.

[16]. Maisch T. Anti-microbial photodynamic therapy: useful in the future? Lasers Med Sci. 2007;22:83-91. 
[17]. Hyun-Hwa Song, Jae-Kwan Lee, Heung-Sik Um, Beom-Seok Chang, Si-Young Lee,Min-Ku Lee. (2013). Phototoxic effect of blue light on the planktonic and biofilm state of anaerobic periodontal pathogens. J Periodontal Implant Sci. 43(2): 72-78.

[18]. Nikolaos S. Soukos, $1,{ }^{*}$ Sovanda Som, 1 Abraham D. Abernethy, 1 Karriann Ruggiero, 1 Joshua Dunham, 1 Chul Lee, 2 Apostolos G. Doukas, 3 and J. Max Goodson4.2005. Phototargeting Oral Black-Pigmented Bacteria. American society of microbiology.

[19]. Feuerstein O, Persman N, Weiss EI. Phototoxic effect of visible light on Porphyromonas gingivalis and Fusobacterium nucleatum: an in vitro study. Photochem Photobiol. 2004;80:412-415.

[20]. Feuerstein O, Ginsburg I, Dayan E, Veler D, Weiss EI. Mechanism of visible light phototoxicity on Porphyromonas gingivalis and Fusobacterium nucleatum. Photochem Photobiol. 2005;81:1186-1189.

[21]. Olsvik, B. and F. C. Tenover (1993) Tetracycline resistance in periodontal pathogens. Clin. Infect. Dis. 16, 3 10-3 13.

[22]. Ferez, M., A. D. Haffajee, C. Goncalves, K. A. Allard, S. Sorn, C. Smith, J. M. Goodson and S. S. Socransky (1999) Systemic doxycycline administration in the treatment of periodontal infections. II. Effect on antibiotic resistance of subgingival species. J. Clin. Periodontol. 26, 784-792.

[23]. Marsh, P. D. and D. J. Bradshaw (1999) Microbial community aspects of dental plaque. In Dental Plaque Revisited: Oral Biofilms in Health and Disease (Edited by H. Newman and M. Wilson), pp. 237-253. Bioline, Cardiff, UK.

[24]. Perry DA. Plaque control for the periodontal patient. In: Newman MG, Takei HH, Klokkevold PR, Carranza FA, Editor. Carranza's Clinical Periodontology. 10th ed. St. Louis, Missouri:Saunders; 2006. p. 728.

[25]. Parwani SR, Parwani RN, Chitnis P J, Dadlani HP, Sai Prasad SV. Comparative evaluation of anti-plaque efficacy of herbal and $0.2 \%$ chlorhexidine gluconate mouthwash in a 4-day plaque re-growth study. J Indian Soc Periodontol 2013; 17:72-7.

[26]. Addy M. The use of antiseptics in periodontal therapy. In: Lindhe J, Karring T, Lang NP, editors. Clinical Periodontology and Implant Dentistry. 4thed. New Delhi: Jaypee Brothers Medical Publishers (P) Ltd.; 2003. p. 464.

[27]. Lyle DM. Chemotherapeutics and Topical Delivery Systems. In: Wilkins EM, editor. Clinical Practice of the Dental Hygienist. 9th ed. Philadelphia: Wolters Kluwer Company; 2005.p. 439.

[28]. Mahesh CP, Peter S. Plaque Control. In: Peter S, editor. Essentials of Preventive and Community Dentistry. 2nd ed. New Delhi: Arya Publishing House; 2003. p. 447.

[29]. Peter M. Loomer, Gary C. Armitage. periodontics: medicine, surgery, and implants. 1st ed. elsevier mosby: St. Louis, Missouri; 2004

[30]. Martin Addy and John Moran. Clinical Periodontology and Implant Dentistry. 5th Ed. Blackell Munksgaard : Oxford ; 2008.

[31]. Bala Subramanya Goutham, Kavita Manchanda, Avishek De Sarkar, Ravi Prakash, Kunal Jha, and Shafaat Mohammed. (2013). Efficacy of two commercially available Oral Rinses - Chlorhexidine and Listerine on Plaque and Gingivitis - A Comparative Study. J Int Oral Health 5(4): 56-61.

[32]. Feuerstein O, Persman N, Weiss EI. Phototoxic effect of visible light on Porphyromonas gingivalis and Fusobacterium nucleatum: an in vitro study. hotochem Photobiol. 2004;80:412-415.

(Table 1)Median Value of CHX mouthwash inhibition zone against Aggregatibacter actinomycetemcomitans with different light exposure time

\begin{tabular}{|c|c|c|c|c|c|c|}
\hline $\begin{array}{l}\text { Time } \\
\text { (Sec.) }\end{array}$ & Median & Mean Rank & $X^{2}$ & d.f & p-value & Significance \\
\hline Control (zero) & 6 & 16.46 & \multirow{4}{*}{8.107} & \multirow{4}{*}{3} & \multirow{4}{*}{0.044} & \multirow{4}{*}{ S } \\
\hline 20 & 7.5 & 22.54 & & & & \\
\hline 40 & 8.5 & 26.96 & & & & \\
\hline 60 & 10 & 32.04 & & & & \\
\hline
\end{tabular}

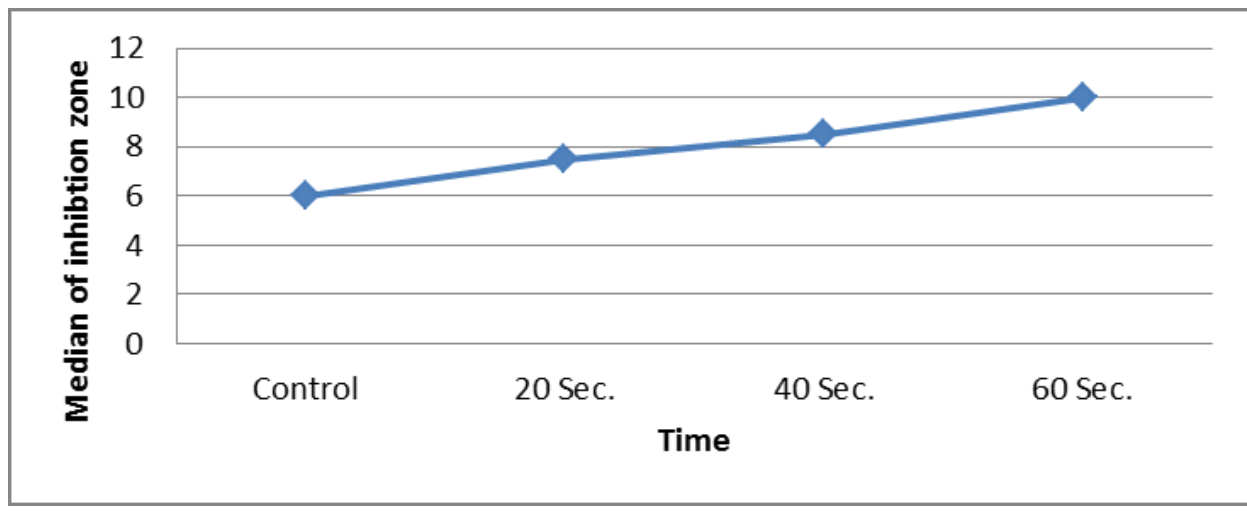

Fig 4: Time of light exposure VS. The CHX mouthwash inhibition zone against Aggregatibacter actinomycetemcomitans 


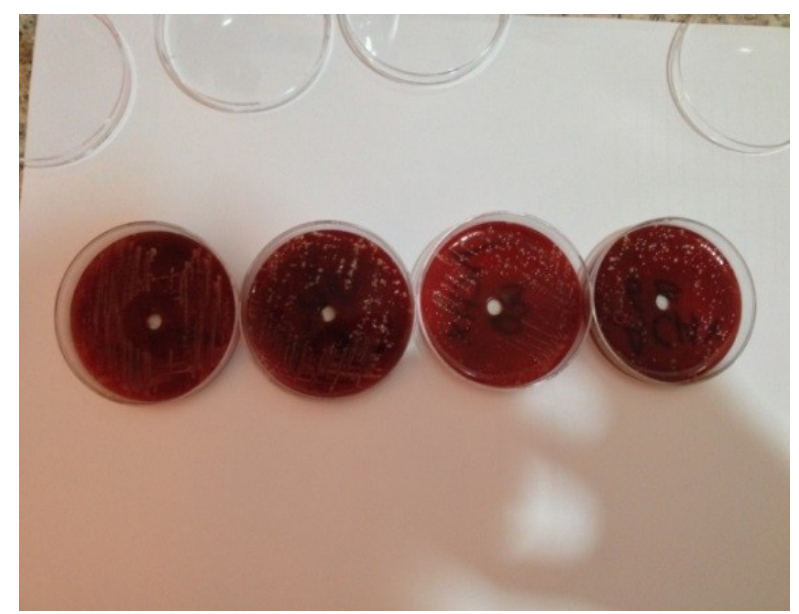

Fig 5: the gradual increase(from left to right) in the inhibition zone of CHX as we proceed from zero/seconds(without) light exposure to 60/seconds of light exposure.
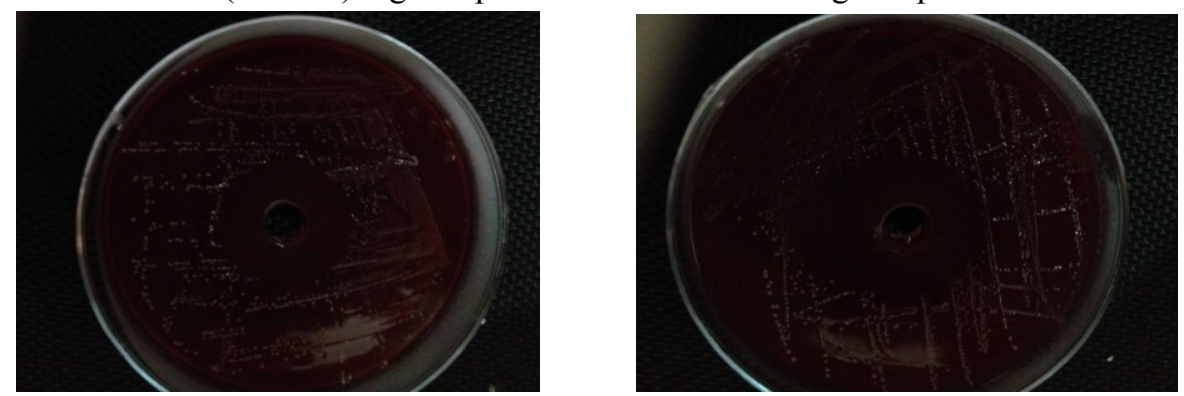

Fig 6: A(left):inhibition zone in a plate of Aggregatibacter actinomycetemcomitans without light exposure. B(right): inhibition zone in a plate of Aggregatibacter actinomycetemcomitans with 60 seconds of light exposure.

(Table 2)Intergroup comparison between CHX mouthwash inhibition zones against Aggregatibacter actinomycetemcomitans of each two groups of light exposure time

\begin{tabular}{|l|l|l|l|}
\hline Time (Sec.) & Mann-Whitney U test & Z-test & p-value \\
\hline Control vs. $20 \mathrm{sec}$. & 49.5 & -1.306 & 0.191 (NS) \\
\hline Control vs. $40 \mathrm{sec}$. & 39 & -1.913 & 0.056 (NS) \\
\hline Control vs. $60 \mathrm{sec}$. & 31 & -2.377 & 0.017 (S) \\
\hline 20 sec. vs. 40 sec. & 56.5 & -0.9 & $0.368(\mathrm{NS})$ \\
\hline 20 sec. vs. 60 sec. & 41.5 & -1.769 & $0.077(\mathrm{NS})$ \\
\hline 40 sec. vs. 60 sec. & 53 & -1.104 & $0.270(\mathrm{NS})$ \\
\hline
\end{tabular}

(Table 3)Median Value of CHX mouthwash inhibition zone against Porphyromonas gingivalis with different light exposure time

\begin{tabular}{|c|c|c|c|c|c|c|}
\hline $\begin{array}{l}\text { Time } \\
\text { (Sec.) }\end{array}$ & Median & Mean Rank & $\mathrm{X}^{2}$ & d.f & p-value & Significance \\
\hline Control (zero) & 7 & 17.54 & \multirow{4}{*}{7.712} & \multirow{4}{*}{3} & \multirow{4}{*}{0.05} & \multirow{4}{*}{ S } \\
\hline 20 & 7.25 & 21.63 & & & & \\
\hline 40 & 8 & 26.25 & & & & \\
\hline 60 & 10.5 & 32.58 & & & & \\
\hline
\end{tabular}




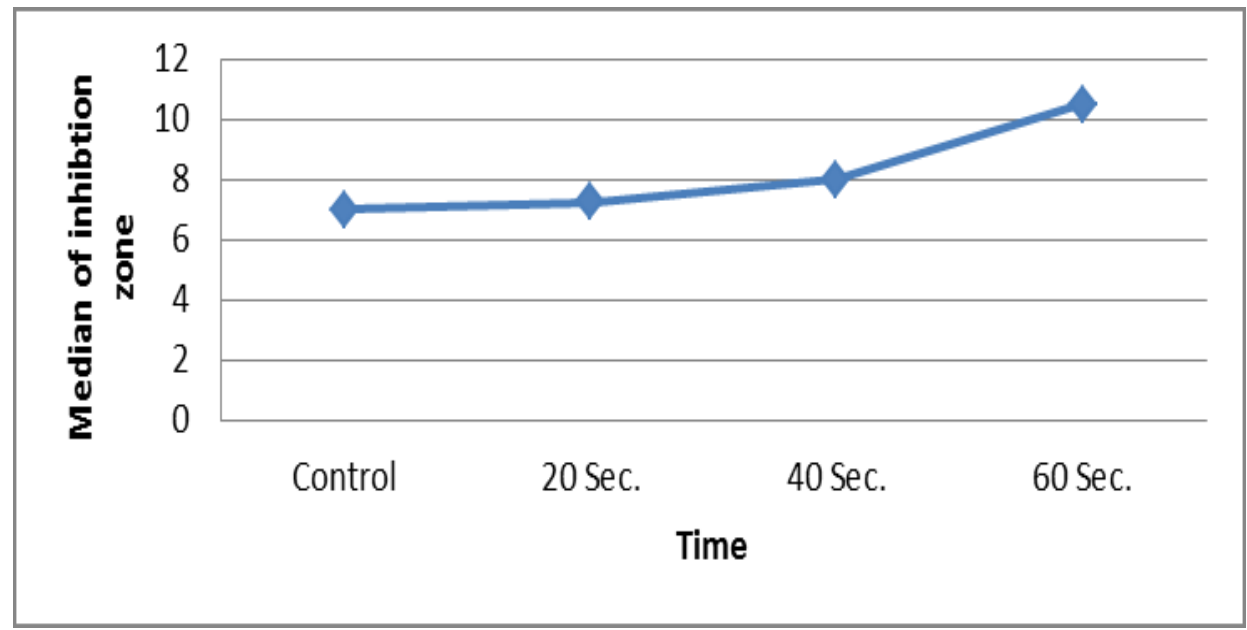

Fig 7: Time of light exposure VS. The CHX mouthwash inhibition zone against Porphyromonas gingivalis.
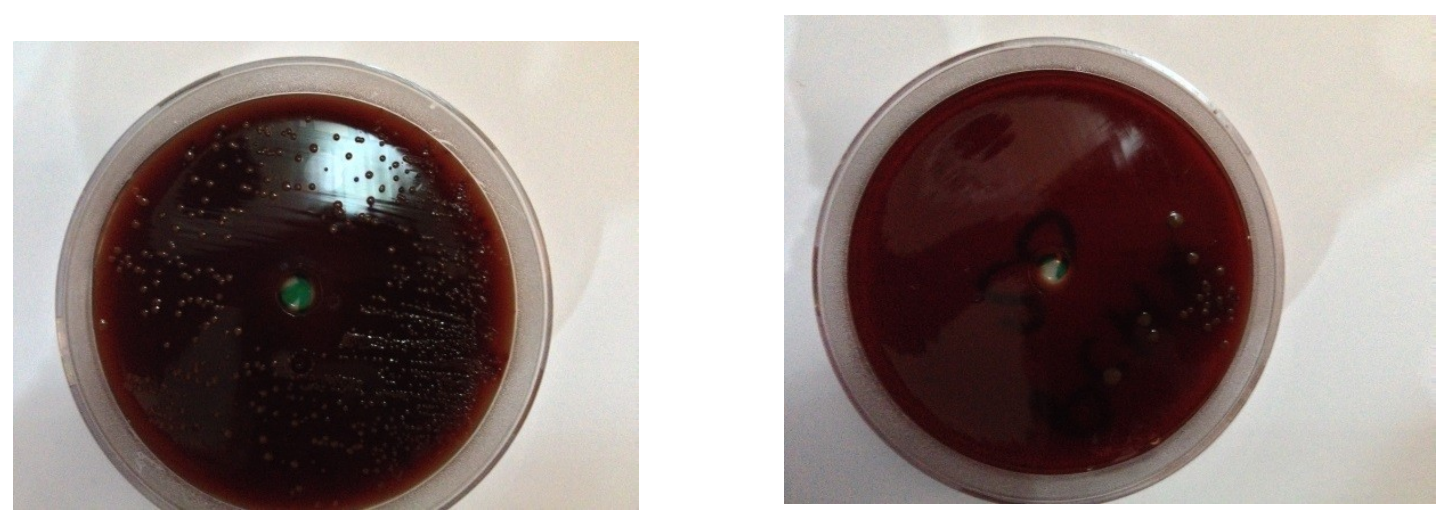

Fig 8: A(left):inhibition zone in a plate of Porphyromonas gingivalis without light exposure.

$\mathrm{B}$ (right): inhibition zone in a plate of Porphyromonas gingivalis with 60 seconds of light exposure.

(Table4)Intergroup comparison between CHX mouthwash inhibition zones against Porphyromonas gingivalis of each two groups of light exposure time

\begin{tabular}{|l|l|l|l|}
\hline Time (Sec.) & Mann-Whitney U test & Z-test & p-value \\
\hline Control vs. 20 sec. & 57 & -0.870 & $0.384(\mathrm{NS})$ \\
\hline Control vs. 40 sec. & 46.5 & -1.482 & $0.138(\mathrm{NS})$ \\
\hline Control vs. 60 sec. & 29 & -2.493 & $0.013(\mathrm{~S})$ \\
\hline 20 sec. vs. 40 sec. & 55.5 & -0.957 & $0.339(\mathrm{NS})$ \\
\hline 20 sec. vs. 60 sec. & 39 & -1.912 & $0.056(\mathrm{NS})$ \\
\hline 40 sec. vs. 60 sec. & 51 & -1.224 & $0.221(\mathrm{NS})$ \\
\hline
\end{tabular}

